\title{
Propagation analysis of electromagnetic waves between the helium and proton gyrofrequencies in the low-altitude auroral zone
}

\author{
Ondřej Santolík \\ Faculty of Mathematics and Physics, Charles University, Prague, Czech Republic \\ Michel Parrot \\ Laboratoire de Physique et Chimie de l'Environnement, Centre National de la Recherche Scientifique, Orléans \\ France
}

\begin{abstract}
We present a statistical study of electromagnetic emissions with a lower frequency limitation observed in a broadband below the local proton gyrofrequency. An extensive data set recorded by the low-orbiting Freja satellite is examined. The emissions occur outside the plasmasphere, mainly at auroral latitudes. The maximum wave power is observed before the local noon, and no events have been detected during the local night. In the majority of cases we can assume the presence of a single plane wave. Further analysis shows a nearly circular and right-handed polarization. The statistics of wave vector directions reveal a downward and nearly field aligned propagation. An experimental estimation of the phase velocity modulus is in rough agreement with the cold plasma theory. The origin of the emissions could be possibly explained by the tunnelling of the downgoing ELF hiss below the two-ion cross-over frequency.
\end{abstract}

\section{Introduction}

ELF waves below the local proton gyrofrequency $f_{\mathrm{H}^{+}}$were frequently observed in the past from several spacecraft at high latitudes. Narrow-banded emissions have been seen on Injun 5 [Gurnett and Frank, 1972], on S3-3 [Temerin and Lysak, 1984], on Aureol 3 [Rauch et al., 1985], on ISIS 1 and 2 [Saito et al., 1987], on Viking [Gustafsson et al., 1990], and also on Freja [Oscarsson et al., 1997]. The frequencies of the emissions $(100-500 \mathrm{~Hz})$ are mainly between the proton and the helium gyrofrequencies but they have been also detected below by Gustafsson et al. [1990]. A statistical study by Saito et al. [1987] shows that the emissions mostly occurred during evening and premidnight hours. Temerin and Lysak [1984] found that these waves are generated by inverted- $\mathrm{V}$ electrons in the auroral acceleration region. This generation mechanism leads to downgoing waves with phase velocities nearly perpendicular to the direction of the static magnetic field $\mathbf{B}_{0}$. Several studies have dealt with numerical calculations of growth rates for this instability [Gustafsson et al., 1990; Oscarsson and Rönnmark, 1990; Santolik and Parrot, 1996; Lund and LaBelle, 1997; Oscarsson et al., 1997]. The plasma models derived from measured electron distributions give generally too low growth rates, but in a distant generation region the wave growth could be sufficient to explain the observed waves. Analysis of narrow-banded cases of Oscarsson and Rönnmark [1990] and Oscarsson et al. [1997] gives a good correspondence of the measured wave polarization to the predicted perpendicular propagation.

An important class of auroral emissions below $f_{\mathrm{H}^{+}}$is not strictly narrow-banded. The ELF waves can occupy a large fraction of the frequency interval between the local helium gy-

Copyright 1998 by the American Geophysical Union.

Paper number $98 \mathrm{JA} 01386$.

0148-0227/98/98JA-01386\$09.00 rofrequency $f_{\mathrm{He}^{+}}$and $f_{\mathrm{H}^{+}}$[Lefeuvre et al., 1992]. The emissions are very often associated with the electromagnetic ELF hiss above $f_{\mathrm{H}^{+}}$(O. Santolík and M. Parrot, Case studies on the wave propagation and polarization of ELF emissions observed by Freja around the local proton gyrofrequency, submitted to Journal of Geophysical Research, 1998, hereinafter referred to as Santolík and Parrot, submitted manuscript, 1998). There could also be no gap in frequency spectra between the ELF waves below $f_{\mathrm{H}^{+}}$, and the hiss emissions [e.g., Lefeuvre et al., 1992]. However, a common feature of all these emissions seems to be a lower frequency limitation of both electric and magnetic components. This lower frequency limit is often located near the local $f_{\mathrm{He}}$, but the exact value of $f_{\mathrm{He}^{+}}$seldom corresponds to any structure in the wave spectra. The ELF emissions extend sometimes well below the local $f_{\mathrm{He}}+$ and often the lower limit is shifted above $f_{\mathrm{He}^{+}}$. In cases analyzed by Lefeuvre et al. [1992] and Rauch et al. [1985], the direction of the wave vector $\mathbf{k}$ was found nearly parallel to $\mathbf{B}_{0}$ below $f_{\mathrm{H}^{+}}$, contrary to the direct output of the above mentioned generation mechanism. They also reported a right-hand-polarized propagation mode.

Theoretical wave mode structure below $f_{\mathrm{H}}+$ strongly depends on the ion composition of the plasma medium. The linear theory of a homogeneous, cold and multicomponent plasma [Smith and Brice, 1964] predicts two wave modes below the gyrofrequency of the heaviest ion: the fast compressional Alfvén mode and the slow shear Alfvén mode. The slow mode has left-hand $(\mathrm{L})$ polarization with respect to $\mathbf{B}_{0}$, and an oblique resonance for nearly perpendicular propagation. The fast mode is right-handed $(R)$ and extraordinary $(\mathrm{X})$. Below each gyrofrequency of additional lighter ion species three new characteristic frequencies appear, the two-ion hybrid frequency $f_{\mathrm{ih}}$, the $\mathrm{L}$ mode cutoff frequency $f_{\text {cL }}$ and the cross-over frequency $f_{\text {co }}$. Suppose, e.g., a singleionized hydrogen-oxygen plasma. Between $f_{\mathrm{O}}+$ and $f_{\mathrm{ih}}$, only the R-X mode can propagate. Between $f_{\text {ih }}$ and $f_{\mathrm{cL}}$, the $\mathrm{R}$ mode has an oblique resonance and the extraordinary perpendicular 
propagation becomes impossible. Between $f_{\mathrm{cL}}$ and $f_{\text {co }}$, two modes exist, fast $\mathrm{L}-\mathrm{X}$ and slow $\mathrm{R}$ with an oblique resonance. Between $f_{\text {co }}$ and $f_{\mathrm{H}^{+}}$, the fast mode changes to $\mathrm{R}-\mathrm{X}$ and the slow mode has the $\mathrm{L}$ polarization and an oblique resonance. The $\mathrm{L}$ polarization appears only between the cutoff at $f_{\mathrm{cL}}$ and the principal resonance at $f_{\mathrm{H}^{+}}$, whereas the $\mathrm{R}$ polarized mode propagates in all the frequency interval. At $f_{\text {co }}$ the polarization sense is exchanged, the fast mode manifests isotropic properties, and the phase velocity of both modes coincides only for the parallel propagation. The slow mode with the oblique resonance between $f_{\mathrm{ih}}$ and $f_{\mathrm{H}^{+}}$is sometimes referred to as electro-magnetic hydrogen cyclotron waves (e.g., by Lund and LaBelle [1997]). Similar mode structure also appears below the gyrofrequencies of other ion species (e.g. Helium), if present.

The aim of the present paper is to examine the propagation mode, direction and other characteristics of electromagnetic waves between $f_{\mathrm{He}^{+}}$and $f_{\mathrm{H}^{+}}$. We deal with a data set collected by the low-orbiting Freja satellite. In section 2 we present these data. We also briefly introduce various analysis methods used later in the paper. In section 3, a case study is shown (Freja observation on April 8, 1995) . Results of a statistical analysis using numerous cases are presented in section 4 . Concise conclusions with a short discussion are given in section 5 .

\section{Data Processing}

The Freja satellite operated between October 1992 and October 1996 on a low-altitude orbit with an apogee of $1756 \mathrm{~km}$ and an inclination of $63^{\circ}$. The mission was devoted mainly to high-altitude studies (for details, see Space Science Reviews, 70, (3/4), 1994). The wave experiment F4 [Holback et al., 1994] measured waveforms of electric and magnetic fields and plasma density in the following frequency ranges: dc, $L F$ (up to $2 \mathrm{kHz}$ ), MF (up to $16 \mathrm{kHz}$ ), and HF (up to $4 \mathrm{MHz}$ ). In this study we use the LF band, where four programmable data channels are available. We have selected time intervals where these channels transmit three orthogonal magnetic components, and one electric component measured by a spinning antenna. In order to achieve necessary coordinate transformations and to determine the gyrofrequencies, we also use the dc magnetic field provided by the F2 fluxgate magnetometer [Freja Magnetic Field Experiment Team, 1994]. Our data processing takes into account a noncontinuous way of F4 data recording: the digitalized waveforms are provided in snapshots, each of them containing a predefined number of samples. As between each two adjacent snapshots there are relatively long data gaps, we never mix the samples from different snapshots during the spectral analysis.

Several different methods are used to determine wave characteristics in this study. Some of them are based on the assumption about the presence of a single plane wave. The direction where the variance of the wave magnetic field is minimum then defines the $\mathbf{k}$ direction. Prior to the analysis, we transform the magnetic components to an orthogonal frame of reference linked to the Earth's magnetic field $\mathbf{B}_{0}$ : the $z$ axis lies along $\mathbf{B}_{0}$, the $x$ axis points towards the direction of decreasing magnetic latitude in the local magnetic meridian plane, and the $y$ axis completes the orthogonal set. In this system, the $\mathbf{k}$ direction is characterized by the polar angle $\theta$ and the azimuthal angle $\phi$ (for illustration see Figure 1). The transformed field components are then subjected to the spectral analysis and the spectral matrix $S_{i j}(f)=\left\langle b_{i}(f) b_{j}^{*}(f)\right\rangle$ is calculated using the Fourier transformed magnetic field components $b_{i}, i=1 . .3$ for each frequency $f$. We use three independent plane wave methods

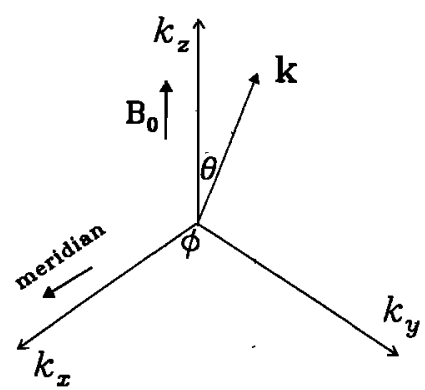

Figure 1. Spherical coordinates used to describe the wavevector direction.

to determine the $\mathbf{k}$ direction. The method of Means [1972] is based on the imaginary part of the spectral matrix; the method of McPherron et al. [1972] uses eigenvector analysis of the real part of the spectral matrix, and the method of Samson and Olson [1980] works with a state vector obtained from the first row of the complex spectral matrix. The determination of the sense of polarization for quasi-parallel waves is based on the sign of the imaginary part of the cross-spectrum between the $B_{x}$ and $B_{y}$ components [Lefeuvre et al., 1986]. If this sign is positive, the waves are right-hand polarized, if it is negative, the waves are left-hand polarized. Note, that we consider the sense of polarization with respect to $\mathbf{B}_{0}$, rather than $\mathbf{k}$, according to the convention often adopted for waves in magnetized plasmas [e.g., Stix, 1992]. The degree of polarization is directly estimated from the spectral matrix of the three magnetic components [Samson and Olson, 1980]. If the degree of polarization has a value of 1 , the wave field is absolutely coherent. This corresponds to the presence of a single plane wave with a single mode. If the wave field is more complex, the degree of polarization decreases [Pinçon et al., 1992]. The method of McPherron et al. [1972] provides an alternative definition of the polarization degree. The ellipticity is estimated using the plane wave hypothesis [Samson and Olson, 1980]. Note that we use the classical Fourier approach, and we suppose a stationary signal during time intervals of the spectral analysis. These intervals are, however, very short (typically fractions of a second) and, additionally, our signals are often rather broadbanded. In such a case, the classical approach is more appropriate than alternative methods, like wave-step analysis in Anderson et al. [1996].

All the methods listed above use only the measurement of the wave magnetic field. This implies an obvious ambiguity of the resulting $\mathbf{k}$ direction: we cannot distinguish between two antiparallel vectors. If we obtain a direction $(\theta, \phi)$ the wave could also propagate at $\left(180^{\circ}-\theta, 180^{\circ}+\phi\right)$ or as a superposition of arbitrary percentage of both these possibilities. The symmetry imposed by the terrestrial magnetic field divides the $4 \pi$ solid angle into two hemispheres separated by the plane $k_{x}-k_{y}$ in Figure 1. In the northern auroral region, the propagation with $k_{z}>0$ corresponds to downgoing waves. The important point is that the direction of Poynting flux always lies in the same hemisphere as the direction of $\mathbf{k}$. We must therefore include also the measurement of the electric field to distinguish between upand downgoing waves [Lefeuvre and Parrot, 1984]. Using again the plane wave hypothesis, a simplified approach can be adopted, and for the Freja wave data, we have developed a procedure based on a measurement of a single spinning electric antenna (Santolik and Parrot, submitted manuscript, 1998). Using the three-axial magnetic field, a component $B_{p}$ is calculated. $B_{p}$ 
lays in the $(x, y)$ plane and is both perpendicular to the $z$ axis and to the direction of the electric antenna. Note that $B_{p}$ can be calculated only if the direction of the electric antenna differs from $\mathbf{B}_{0}$. Then, we calculate the cross-spectrum $S_{B E}$ between $B_{p}$ and the electric component. The sign of the real part of $S_{B E}$ finally determines the sign of the $z$ component of the Poynting vector. Using an estimate $\delta \Re S_{B E}$ of the experimental error on this cross spectrum, a direction indicator $\Re S_{B E} / \delta \Re S_{B E}$ can be defined. If the direction indicator is negative, wave propagates downward. Positive sign similarly corresponds to up-going waves. The absolute value gives an idea about reliability of the result.

When the plane wave hypothesis is not valid, the determination of the wave distribution function (WDF) is an appropriate approach to describe the wave field. The WDF specifies the distribution of wave-energy density with respect to the frequency and the wave-normal direction [Storey and Lefeuvre, 1974, 1979, 1980]. It can be obtained using the data of several magnetic and electric antennae. The upgoing and downgoing waves are distinguished by the WDF analysis in a natural way. Examples of the wave analysis using the WDF can be found, e.g., in Parrot and Lefeuvre [1986] and Lefeuvre et al. [1992]. For a slightly different approach, see Oscarsson et al. [1997, and references therein]. To resolve the inverse problem of the WDF reconstruction, we use a maximum entropy model optimized by a nonlinear least squares method to fit the data [Lefeuvre and Delannoy, 1979; Delannoy and Lefeuvre, 1986]. For the WDF treatment under the cold plasma approximation we need to know the plasma frequency, the electron gyrofrequency and the ion composition. The plasma frequency $\left(f_{p}\right)$ can be obtained from the position of natural narrow-banded emissions of Langmuir waves sometimes seen in HF spectrograms. The second possibility is to use the density variation measured by the Langmuir probe, when it is available. The electron gyrofrequency is directly obtained from the measurement of the dc magnetic field by the F2 fluxgate magnetometer. The ion composition is estimated using $f_{p}$ and the lower hybrid frequency $\left(f_{l h}\right)$ inferred from a sharp cutoff of electrostatic waves in the MF spectrograms. Both characteristic frequencies correspond to local electrostatic emissions, and the local ion composition is obtained supposing a single-ionized $\mathrm{O}^{+}-\mathrm{H}^{+}$plasma. Obviously, some helium fraction could also be present. Neglecting it, we however do not strongly perturb the propagation characteristics between $f_{\mathrm{H}^{+}}$and $f_{\mathrm{He}^{+}}$.

\section{Detailed Study of One Event}

As an example of waves between the hydrogen and helium gyrofrequencies we have chosen a Freja observation on April 8, 1995. Between 1547 and 1607 UT the satellite passed over the Prince Albert receiving station and the F4-LF device measured one electric and three magnetic field components. Waveform data have been recorded in 8-s cycles with $3 \mathrm{~s}$ of data recording separated by 5 -s data gaps. Figure 2 shows spectrograms of magnetic and electric field in ELF range recorded during this passage. The magnetic component is chosen to be parallel to the satellite spin axis, while the single electric component is measured by a spinning antenna. The spin patterns with a period of about $12 \mathrm{~s}$ (about $6 \mathrm{~s}$ of spin period strobed by the 8-s period of waveform snapshot) are therefore present on the electric spectrogram as well as additional dropouts due to instrumental effects. Both spectrograms show a similar overall pattern of relatıvely intense emissions between 1548 and 1555 UT. The waves at higher frequencies can be attributed to the ELF hiss. A less intense hiss emission continues also after 1600 UT. The lower cutoff of the ELF hiss, first explained by Gurnett and Burns [1968], is seen on both spectrograms as a sharp boundary just below the local proton gyrofrequency. At low frequencies, another type of wave phenomena can be observed mainly near 1556 and after 1559 UT. The emissions start at the lowest frequencies, and gradually vanish with increasing frequency. They are better seen in the electric spectrogram where the signal goes up to about $400 \mathrm{~Hz}$, nevertheless the magnetic signature is also detectable below about $200 \mathrm{~Hz}$. These emissions are similar to the kinetic Alfvén waves observed in the auroral region by Wahlund et al. [1994]. Returning to the time interval 1548 $1555 \mathrm{UT}$, we can finally see an intense emission between the local helium and proton gyrofrequencies. The lower cutoff is not as sharply pronounced comparing to the ELF hiss emissions; it is rather diffuse and changes its frequency. The waves are observed on both magnetic and electric spectrograms. The maximum intensity is recorded near 1553 UT. At this moment, the satellite operates in the morning sector of the auroral region at an altitude of $1100 \mathrm{~km}$. A 3-s snapshot of waveform data measured from 1553:05.3 UT will be analyzed in more detail. The waveforms of all field components reveal a strong signal having a period of about $2.5 \mathrm{~ms}$, weakly modulated with irregular longer-period pulsations, and changing the phase in a stochastic manner (not shown).

Spectral analysis of these data has been performed with 4$\mathrm{Hz}$ resolution as an average of $24 \mathrm{FFT}$ estimates. The resulting spectral matrices have served as input data for three independent analysis methods (see section 2). The results are collected in Figure 3. The power spectrum on the top corresponds to the magnetic $B_{z}$ component (parallel to the ambient field $\mathbf{B}_{0}$ ). The ELF hiss is cut off just below $f_{\mathrm{H}^{+}}$. At lower frequencies, we see a broadband emission with a maximum around $400 \mathrm{~Hz}$, extending down to $f_{\mathrm{He}^{+}}$. At lowest frequencies below $100 \mathrm{~Hz}$, the wave power again rises. The next panel of Figure 3 gives the values of polarization degree $P$ obtained by the methods of McPherron et al. [1972] and Samson and Olson [1980]. Both curves give lower degree of polarization in the case of the ELF hiss above $f_{\mathrm{H}^{+}}$. The value is about 0.6 for both methods, with some fluctuations below this value in the case of $M c$ Pherron et al. [1972]. A very pronounced decrease is observed in a narrow interval of $20 \mathrm{~Hz}$ below $f_{\mathrm{H}^{+}}$, followed by higher values between 320 and $520 \mathrm{~Hz}$ ( $P \approx 0.9$ from McPherron et al. [1972] and about $P \approx 0.8$ from Samson and Olson [1980]). The lower frequency part of this emission between $f_{\mathrm{He}^{+}}$and about 320 $\mathrm{Hz}$ manifests again lower $P$ values, similarly as for the ELF hiss. Below $100 \mathrm{~Hz}$ the polarization degree slightly increases. The interpretation can be done in terms of testing the hypothesis of a single plane wave. For instance, in the frequency interval between 320 and $520 \mathrm{~Hz}$, the wave field approaches the behavior of a single plane. The decrease of wave polarization just below $f_{\mathrm{H}^{+}}$is a very common feature. It is present in nearly all cases recorded in the auroral region. This feature can be explained by the presence of complex wave fields (e.g., two simultaneous wave modes) in the frequency interval between the $L$-mode cutoff at $f_{\mathrm{cL}}$ and $f_{\mathrm{H}^{+}}$.

The results on the ellipticity $E$ are plotted in the following panel of Figure 3. The polarization is circular in the major part of the frequency interval, with an important exception of waves below $100 \mathrm{~Hz}$, where the polarization is nearly linear. The frequency dependence of the $\mathbf{k}$ direction is presented in the next two panels of Figure 3. The results have been obtained 


$10^{-9} 10^{-8} \mathrm{~S}_{\mathrm{Bg}}\left(\mathrm{nT}^{2} / \mathrm{Hz}\right)^{10^{-7}} 10^{-6}$
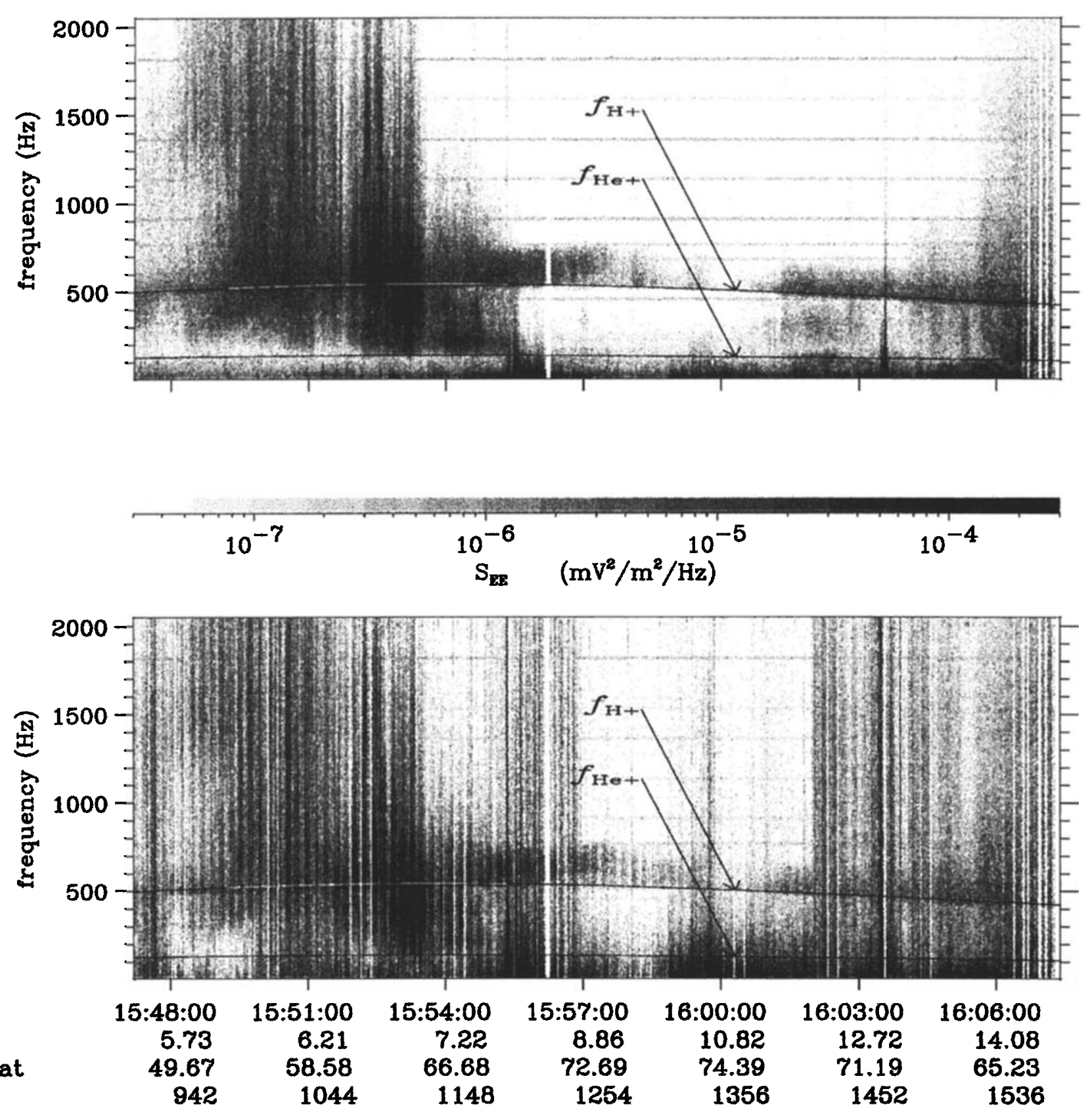

Figure 2. Spectrograms of (top) the magnetic field and (bottom) the electric field as recorded by the Freja F4-LF device on April 8, 1995. The Universal time (UT) on the horizontal axis is completed by the magnetic local time (MLT) in hours, the corrected geomagnetic latitude (CGLat) in degrees, and the altitude (Alt) in kilometers. The local gyrofrequencies $f_{\mathrm{He}^{+}}$and $f_{\mathrm{H}}+$ are overdrawn in both spectrograms.

by three independent methods as shown by different line types. The polar angle $\theta$ is represented in the interval $0^{\circ}-90^{\circ}$, because the methods do not recognize upgoing and downgoing waves. The plot of $\theta$ values is combined with the information on the sense of wave polarization as by Lefeuvre et al. [1992]. For right-hand-polarized waves, $\theta$ is plotted in the bottom half of the panel, and for left-polarized waves in the upper one. If $\theta$ is close to $90^{\circ}$ the left-hand or right-hand polarization has no sense because $\mathbf{B}_{0}$ lies in the polarization plane of the wave magnetic field. The polarization sense is also not defined in the case of nearly linearly polarized waves. Both situations are marked by crosses plotted at $\theta=90^{\circ}$. From Figure 3 it is clearly seen that the right-polarized waves dominate in all the frequency range except in the interval of linear polarization below $100 \mathrm{~Hz}$. The three methods give nearly identical $\theta$ values for the frequencies between 320 and $520 \mathrm{~Hz}$ where the polarization degree is high. 


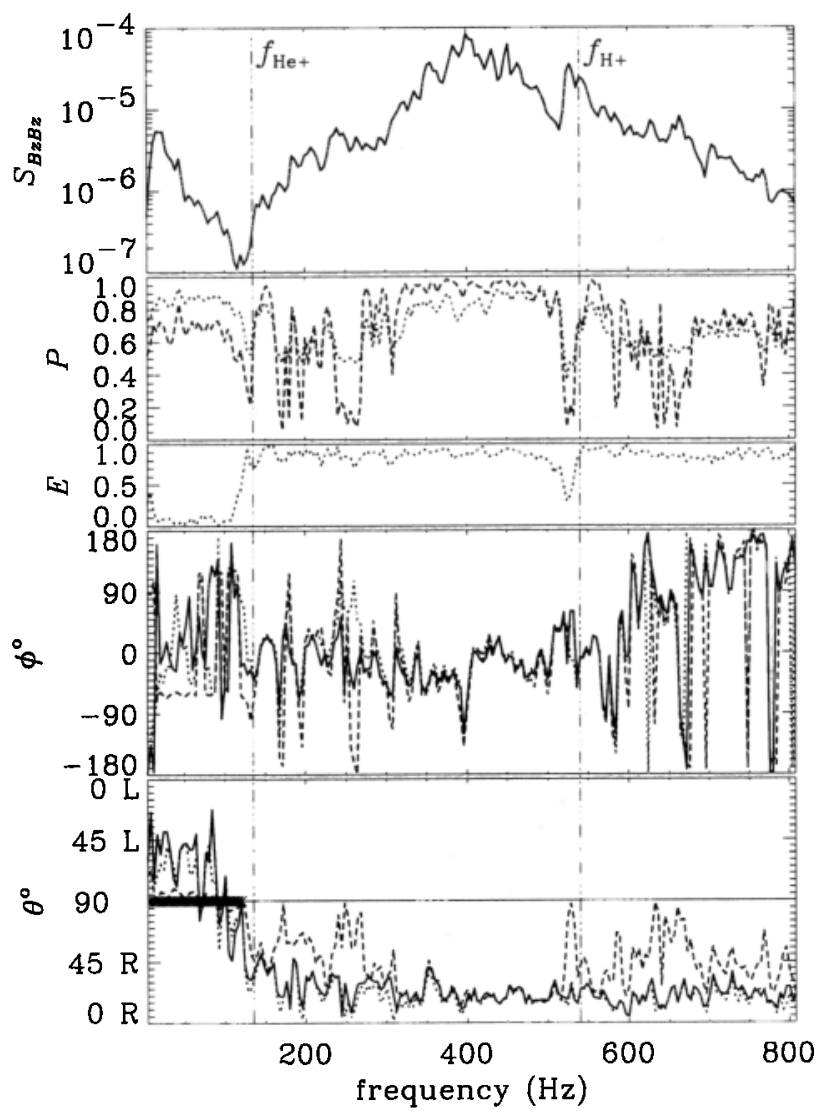

Figure 3. Plane wave analysis of one snapshot of the magnetic field data recorded between 1553:05.3 and 1553:08.3 UT. Several wave parameters are presented as a function of the frequency. From the top: power spectrum $S_{B z B z}$, degree of polarization $P$, ellipticity $E$, azimuth $\phi$, angle deviation $\theta$, and the sense of polarization (see text). Solid lines correspond to the method of Means [1972], dashed lines to the method of McPherron et al. [1972] and dotted lines to the method in Samson and Olson [1980]. The local helium and hydrogen gyrofrequencies are marked by vertical lines at 135 and $540 \mathrm{~Hz}$, respectively.

In the major part of this interval, $\theta$ fluctuates around $20^{\circ}$. Outside this interval the results obtained by the methods of Means [1972] and Samson and Olson [1980] remain near $20^{\circ}$ while the method of $M c$ Pherron et al. [1972] provides higher values. This difference can be explained by the violation of the plane wave hypothesis, as it is also indicated by the decreased polarization degree. Below $100 \mathrm{~Hz}$ the linear wave polarization invalidates the determination of the wave normal direction. Values of the azimuthal angle $\phi$ for the ELF hiss rotate with increasing frequency from $-60^{\circ}$ above $f_{\mathrm{H}+}$ to about $180^{\circ}$ at $800 \mathrm{~Hz}$. The $\phi$ values variate around $0^{\circ}$ in the emission between $f_{\mathrm{He}^{+}}$and $f_{\mathrm{H}+}$. This means that the waves propagate approximately in the plane of the local magnetic meridian. Supposing the waves go down, the obtained $\phi$ values suggest that the wave normal is slightly declined toward lower magnetic latitudes. A large spread of obtained $\phi$ values is probably connected to a relatively small $\theta$ angle.

As follows from the above results, the emission between $f_{\mathrm{He}^{+}}$ and $f_{\mathrm{H}}+$ can be roughly divided into two parts. The frequencies around the main peak of intensity $(320-520 \mathrm{~Hz})$ have polarization properties similar to a single plane wave propagating at $\theta=20^{\circ}$. The lower frequency interval between 120 and 320
$\mathrm{Hz}$ contains less intense signals having lower consistency with the plane wave hypothesis. The three plane wave methods give different estimates of $\mathbf{k}$ direction with $\theta$ varying from about $20^{\circ}$ up to $60^{\circ}-90^{\circ}$. The WDF analysis can accomplish several tasks in this situation. In the lower frequency part it can help to estimate the distribution of wave normals. In the higher frequency part it can verify the results of the plane wave analysis taking into account all accessible components of the spectral matrix. In both bands the processing of the electric field data can help to distinguish the downgoing and upgoing waves. The WDF analysis provides also a good tool to check theoretical predictions on a given wave mode with the observations. To calculate theoretical properties of wave propagation in different directions, we have supposed a cold plasma with $5 \%$ of $\mathrm{H}^{+}$and $95 \%$ of $\mathrm{O}^{+}$, and an electron plasma frequency $f_{\mathrm{p}}=500 \mathrm{kHz}$. These parameters correspond to the electron density measured by the Langmuir probe, and to the observation of an electrostatic emission of the auroral VLF hiss with a lower cutoff at $f_{\mathrm{lh}}$ $\approx 3.5 \mathrm{kHz}$ (not shown). This plasma model can be applied to estimate the two-ion characteristic frequencies and to compare them with the distinctive frequencies of the analysis in Figure 3. The two-ion frequencies are $f_{\mathrm{ih}}=396 \mathrm{~Hz}, f_{\mathrm{cL}}=515 \mathrm{~Hz}$, and

(a)

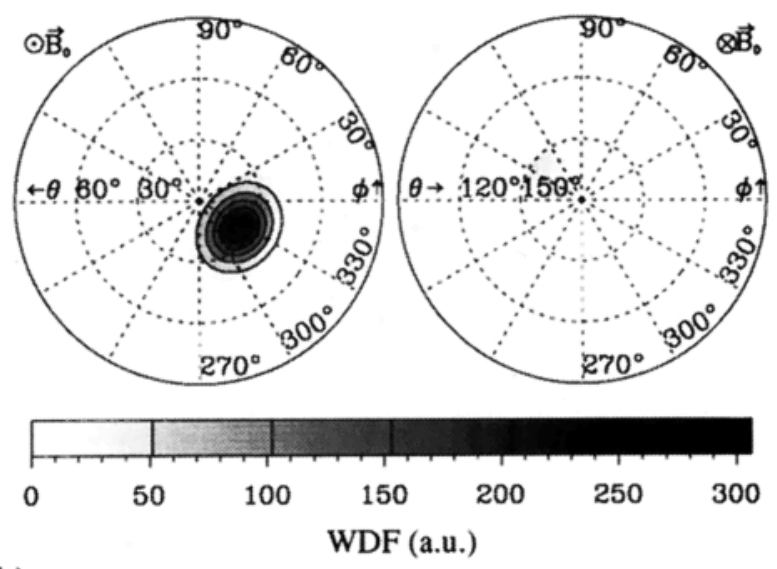

(b)

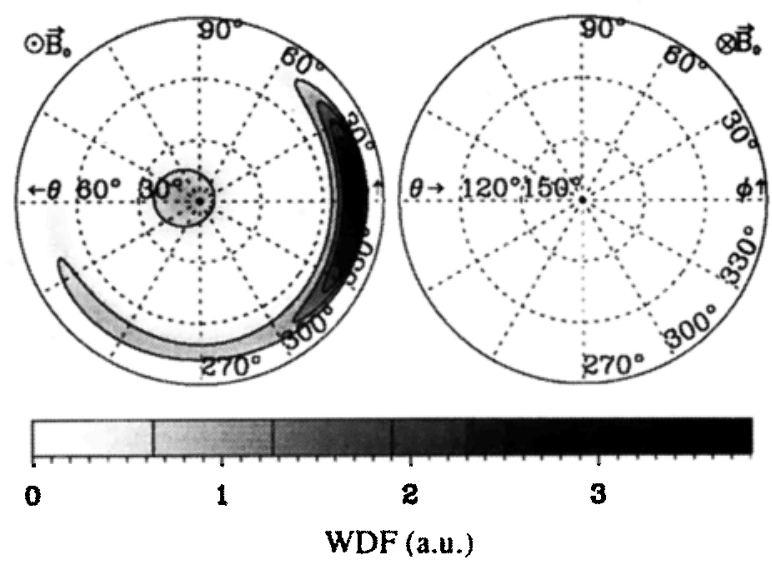

Figure 4. WDF analysis of a part of the data snapshot analyzed in Figure 3. Both electric and magnetic field data have been processed with the MAXENTWDF program [Delannoy and Lefeuvre, 1986]. The WDF in arbitrary units is plotted in $\theta-\phi$ polar diagrams of both hemispheres of (left) downgoing and (right) upgoing waves. The frequency of analysis is (a) $384 \mathrm{~Hz}$ and (b) $224 \mathrm{~Hz}$. 
$f_{\text {co }}=526 \mathrm{~Hz}, f_{\mathrm{H}^{+}}$being $540 \mathrm{~Hz}$. It is shown in Figure 3 that there is no change at $f_{\mathrm{ih}}$, whereas $f_{\mathrm{cL}}$ corresponds to the cutoff frequency in the $B_{z}$ spectrum [Gurnett and Burns, 1968] and to the decrease of the polarization degree and ellipticity.

Figure 4 presents the results of the WDF reconstruction with the above plasma model. Because of the satellite spin period of about $6 \mathrm{~s}$, we cannot use the whole 3-s data snapshot for an analysis including the spinning electric antenna. We have therefore divided the snapshot into 4 parts of $0.75 \mathrm{~s}$, and the analysis has been done on the third part with a frequency resolution of $16 \mathrm{~Hz}$. Possible coupling between the electric antenna and the plasma is another important problem arising from the use of the electric field data. As both antenna rotation and unknown coupling can influence the results, we have first analyzed only the magnetic components, and then both electric and magnetic data. Near the main peak of wave power at $f=384 \mathrm{~Hz}$, the results based on the magnetic components indicate a strong concentration of the wave energy around $\theta \approx 20^{\circ}$ and $\phi$ slightly below $0^{\circ}$ (not shown). The reconstructions using both electric and magnetic data (Figure 4a) reveal that the majority of the wave energy propagates downwards and the peak position does not change. As the results are consistent, the unknown plasma-antenna impedance and the satellite spin have probably no important influence on the WDF reconstruction. We therefore believe the recognition of upgoing and downgoing waves is reliable. The presence of a distinct peak in the WDF shape is consistent with a nearly plane wave. Indeed, the results of the plane wave analysis are confirmed, and the theoretical properties of the right-hand-polarized cold-plasma mode fit well the observations. Figure $4 \mathrm{~b}$ shows the results at $f=224 \mathrm{~Hz}$ presented in a similar form. As expected, the wave field is more complex in the band with a lower polarization degree. The wave power is found near the $\mathbf{B}_{0}$ direction as well as at nearly perpendicular $\theta$ values. The perpendicular waves seem to be concentrated around the direction parallel to the local meridian. We observe a mixture of emissions, possibly of different origin and generated by different mechanisms.

\section{Statistics}

To obtain a more reliable insight into the properties of emissions between $f_{\mathrm{He}^{+}}$and $f_{\mathrm{H}^{+}}$, a set of about 700 time intervals has been collected from about 70 randomly chosen orbits across the whole Freja operational period. The data have been visually selected on electric and magnetic spectrograms as in Figure 2, using the spectral type as the only selection criterion. We have included emissions below $f_{\mathrm{H}^{+}}$with a lower frequency limitation similar to the observations in Rauch et al. [1985], and Lefeuvre et al. [1992], or to the emission before 1555 UT in Figure 2. The width of the frequency band often extends $f_{\mathrm{H}+} / 4$ and there is not always a stop-band below $f_{\mathrm{H}^{+}}$. Some events have a sharp lower cutoff around or below $f_{\mathrm{He}}+$ but a diffuse and fluctuating lower frequency limit is frequent. A plane wave analysis as in Figure 3 has been performed on time intervals of $0.375 \mathrm{~s}$ with a frequency resolution of $32 \mathrm{~Hz}$. The frequency range is restricted to $f_{\mathrm{He}^{+}}-0.8 f_{\mathrm{H}^{+}}$in the major part of our statistical analysis. The upper frequency limit allows us to exclude the interval between $f_{\mathrm{cL}}$ and $f_{\mathrm{H}^{+}}$, where both $\mathrm{R}$ - and L-polarized waves can propagate. For hydrogen fraction less than about $20 \%, f_{\mathrm{cL}}$ is higher than $0.8 f_{\mathrm{H}^{+}}$, and the whole selected inter$\mathrm{val}$ is therefore occupied by a single cold plasma (R-polarized) mode. As shown in the above case study, the polarization parameters are very particular in the narrow interval between $f_{\mathrm{cL}}$ and $f_{\mathrm{H}^{+}}$, comparing to the rest of the frequency band down
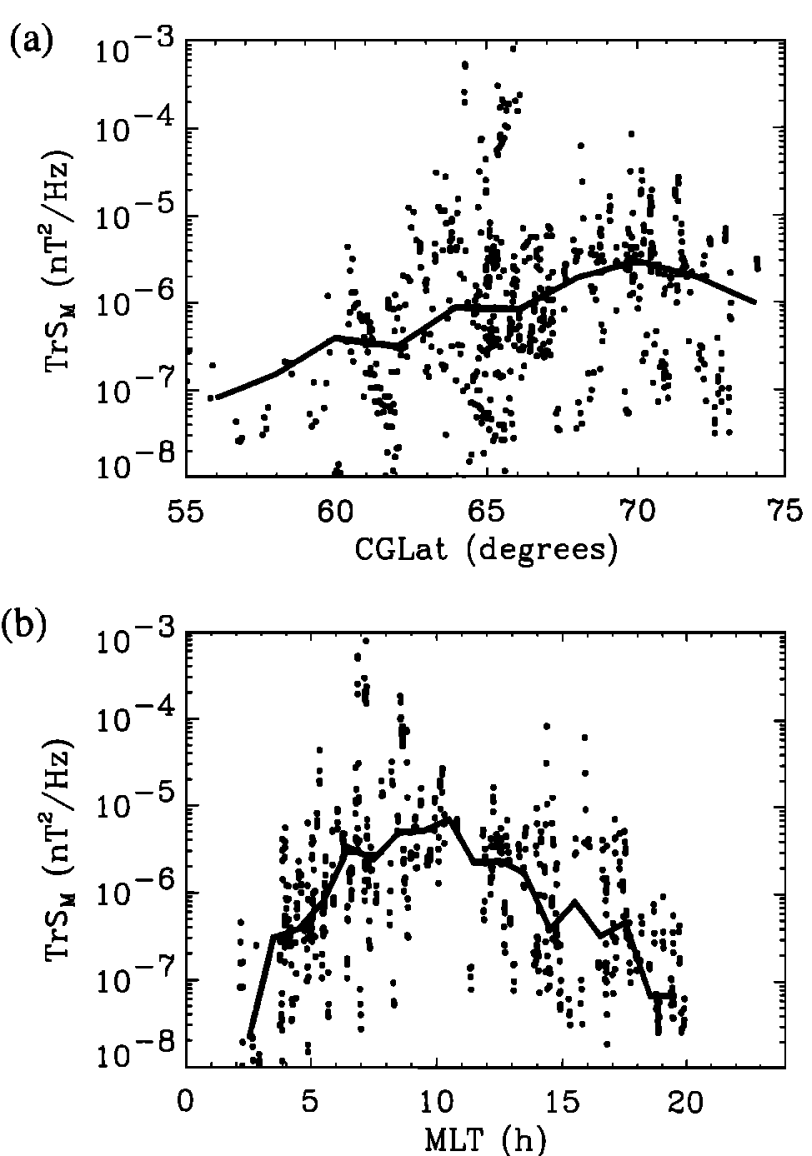

Figure 5. Dependence of the maximum power-spectral densities on (a) CGlat, and (b) MLT. A scatter plot is combined with a solid line indicating the median value calculated in adjacent bins. The bin size is $2^{\circ}$ in CGLat and 1 hour in MLT.

to $f_{\mathrm{He}^{+}}$. It is most likely due to the reflection process of the ELF hiss [Gurnett and Burns, 1968]. Hence we prefer not to mix this phenomenon with the ELF emissions below $f_{\mathrm{cL}}$ and to exclude the waves above $0.8 f_{\mathrm{H}}+$ from the statistics. Naturally, if we examine directly the frequency dependencies of different wave parameters, this exclusion will not be necessary and we will study the whole range between $f_{\mathrm{He}^{+}}$and $f_{\mathrm{H}}+$.

All the events in the statistical set are concentrated around the auroral region at CGLat (corrected geomagnetic latitude) between $60^{\circ}$ and $75^{\circ}$. The upper limit clearly coincides with the maximum magnetic latitude due to the inclination of the satellite orbit. The observations cover a large magnetic local time (MLT) interval from the morning to the evening sector. No events have been found between 20 and 2 hours. The majority of events have been recorded near the apogee of $1756 \mathrm{~km}$. The data set is found at similar latitudes as narrow-banded emissions of Saito et al. [1987]. However, the fact that these emissions have not been found on the night side strongly contrasts with the prevailing eveningside or nightside occurrence of narrowbanded emissions of Saito et al. [1987]. The dependence of the maximum spectral densities on CGLat is shown in Figure 5a. In each event from the data set, the frequency range $f_{\mathrm{He}^{+}}-$ $0.8 f_{\mathrm{H}}+$ has been searched for the maximum spectral density of the magnetic power. The spectral density has been calculated as the sum of power-spectral densities of the three orthogonal magnetic components, i.e., as the trace of the magnetic spectral 
matrix $\operatorname{Tr} \mathrm{S}_{\mathrm{M}}$. The points are scattered in a large range of power densities. The median calculations reveal growing tendency up to CGLat of $70^{\circ}$. The emissions at lower latitudes are less intense. The influence of MLT is more pronounced. Figure 5b shows a scatter plot in MLT- $T r S_{M}$ plane. Spectral densities are higher on the dayside, with a flat peak around 10 hours. In the early moming and late evening the magnetic power rapidly decreases. These quantitative results are well consistent with the absence of nightside observations. Similar results have also been obtained examining all the frequencies between $f_{\mathrm{He}}+$ and $0.8 f_{\mathrm{H}^{+}}$, not only the frequencies of maximum signal intensity (not shown). From the scatterplots in Figure 5 it follows that the values of maximum spectral densities have a large statistical distribution with a maximum occurrence near $2 \times 10^{-6} \mathrm{nT}^{2} / \mathrm{Hz}$ and a median value of $9 \times 10^{-7} \mathrm{nT}^{2} / \mathrm{Hz}$. The high power tail of this distribution extends to $2 \times 10^{-3} \mathrm{nT}^{2} / \mathrm{Hz}$.

Figure 6 gives an answer to the question about the frequency dependence of the wave intensity. A two-dimensional histogram presents the number of events in the frequency- $\operatorname{Tr} S_{M}$ plane. The histogram shows that we often observe a higher spectral density at higher frequencies. Both median and peak of the statistical distribution of $\operatorname{Tr} S_{M}$ are growing functions of the relative frequency. This is strongly connected with the statistics on frequencies where the signal is maximum. If we search for these frequencies in the range $f_{\mathrm{He}^{+}}-0.8 f_{\mathrm{H}^{+}}$, we obtain about one half of events between $0.73 f_{\mathrm{H}^{+}}$and $0.8 f_{\mathrm{H}^{+}}$, i.e. just below the higher limit of the frequency range. The number of cases rapidly decreases with relative frequencies, and only several events have the wave spectrum peaked just above $f_{\mathrm{He}^{+}}$(not shown). The dependence of the maximum signal frequency on CGLat and MLT has been checked but no distinct effects have been noted. The obtained frequency dependence of the wave intensity could be connected to the generation mechanism of the emissions. The emissions below $f_{\mathrm{H}^{+}}$have always a lower frequency limitation and often a characteristic frequency $f_{l}$ of this cutoff can be determined. We believe that the cutoff could also be related to the generation process of these emissions. We have therefore tried to analyse visually the magnetic spectrograms in order to find clear cases with the lower cutoff, and we have succeeded in 33 orbits. As the cutoff characteristics changed during several orbits, more than $40 f_{l}$ values have been estimated. We have classified the estimates into two categories: (1) sharp lower

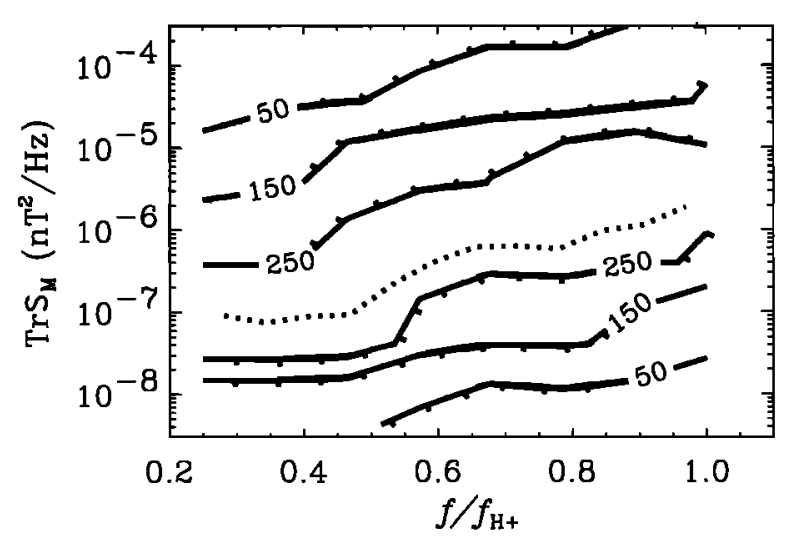

Figure 6. Simultaneous histogram of power-spectral densities and wave frequencies. The frequency- $\operatorname{Tr} S_{M}$ plane is divided into $8 \times 9$ cells. The number of events in each cell is indicated by isolines at $n=50,150$, and 250 . Median values calculated in frequency bins of $0.06 f_{\mathrm{H}^{+}}$are given by a dotted line.

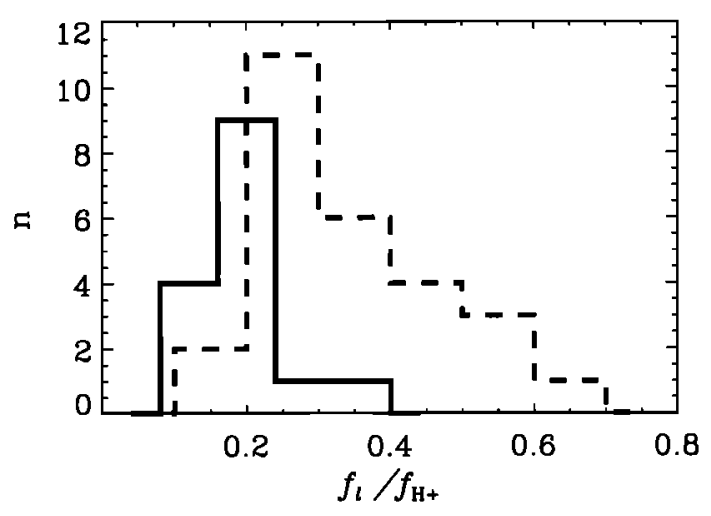

Figure 7. Histograms of lower cutoff frequency $f_{l}$. (solid line) Events with a sharp cutoff (category 1); (dashed line) events with a diffuse lower limitation at a constant frequency (category 2).

cutoff, and (2) diffuse lower limitation at a frequency roughly constant during several minutes. For example, the lower limitation at about $130 \mathrm{~Hz}$ of emissions observed between 1553 and 1555 UT in Figure 2 has been classified in the category 2. Before 1555 UT the lower limitation is highly diffuse and fluctuating. In such cases we have not attempted to estimate $f_{l}$. In Figure 7 we present histograms of the $f_{l}$ positions for both categories separately. The diffuse limitations are spread between $0.15 f_{\mathrm{H}^{+}}$ and $0.7 f_{\mathrm{H}^{+}}$with a median of $0.31 f_{\mathrm{H}^{+}}$. The emissions with a sharp cutoff have $f_{l}$ between $0.1 f_{\mathrm{H}^{+}}$and $0.35 f_{\mathrm{H}}+$ with a median value of $0.18 f_{\mathrm{H}^{+}}$. An interesting point is that these latter events have been found only in the afternoon and evening hours at MLT between 12 and 20 hours. In spite of this, we have observed no clear dependence of $f_{l}$ on the magnetic latitude or local time. We can further see that the helium gyrofrequency $f_{\mathrm{He}^{+}}=0.25 f_{\mathrm{H}^{+}}$is not obviously related to the $f_{l}$ estimates. The label " $f_{\mathrm{He}^{+}}-f_{\mathrm{H}^{+}}$" emissions can therefore serve only to roughly indicate their frequency extent.

Before we proceed with further analysis we will concentrate on the statistics of the degree of polarization, because its value controls the reliability of the plane-wave methods. The example analysis in section 3 has shown that the polarization degree could be strongly dependent on the wave frequency. Statistical

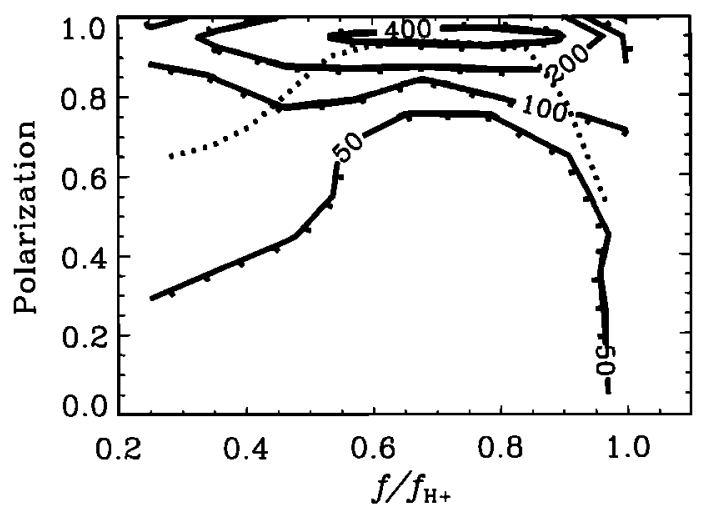

Figure 8. Simultaneous histogram of the polarization degree [McPherron et al., 1972] and the wave frequency. The frequency-polarization plane is divided into $8 \times 10$ cells. The number of events in each cell is indicated by isolines at $n=50$, 100,200 , and 400 . Median values calculated in frequency bins of $0.06 f_{\mathrm{H}}+$ are given by a dotted line. 


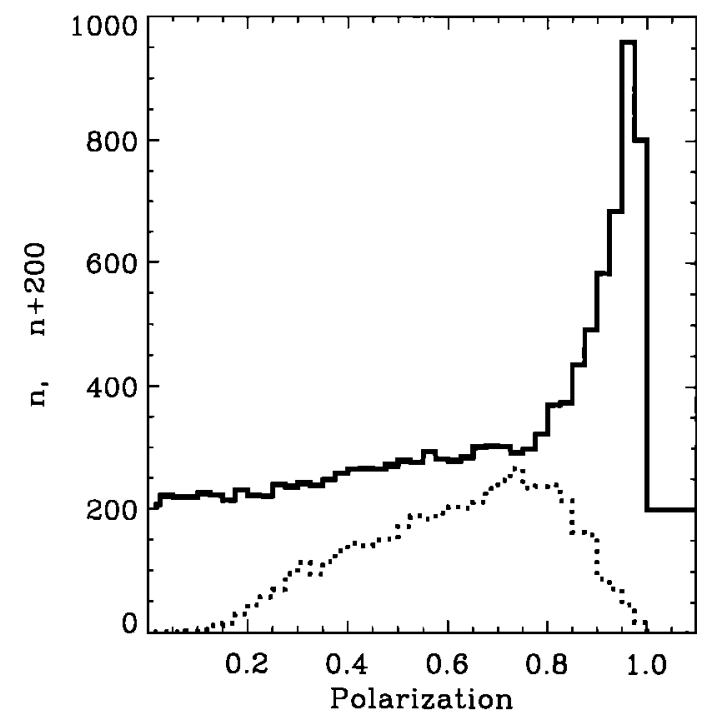

Figure 9. Histogram of the polarization degree. All frequencies between $f_{\mathrm{He}^{+}}$and $0.8 f_{\mathrm{H}}+$ are included. (solid line) The degree of polarization of McPherron et al. [1972]; the number of events in each bin is increased by 200 . (dotted line) Method of Samson and Olson [1980].

results on this dependence are given in Figure 8. We have processed the original data set of about 700 time intervals in the frequency range between $f_{\mathrm{He}^{+}}$and $f_{\mathrm{H}^{+}}$. A two-dimensional histogram in the frequency-polarization plane has been obtained with the method of McPherron et al. [1972]. At frequencies between $0.55 f_{\mathrm{H}^{+}}$and $0.85 f_{\mathrm{H}^{+}}$, nearly all events are concentrated around the main peak of highly polarized waves, with a median of polarization degree above 0.9 . The number of cases with a lower polarization increases below $0.55 f_{\mathrm{H}^{+}}$, and the median goes down to 0.6 . The statistics therefore confirms the increased probability of complex wave fields observed at lower frequencies in section 3 . The statistics similarly shows a very pronounced decrease of the polarization degree at the higher frequency edge of our range. We believe that this is due to the above mentioned phenomena connected with the lower cutoff of ELF hiss. The results further show that the frequency limit of $0.8 f_{\mathrm{H}^{+}}$is more than sufficient to exclude the frequencies contaminated by the hiss reflection process. Figure 9 shows the resulting histogram of the polarization degree, obtained across the reduced frequency interval $f_{\mathrm{He}^{+}}-0.8 f_{\mathrm{H}^{+}}$. Two independent methods have been used as in section 3 . The method of Samson and Olson [1980] gives a large distribution covering nearly all the interval between 0 and 1 (dotted line). The method of McPherron et al. [1972] reveals two distinct parts of the statistical distribution (solid line): a sharply peaked region of nearly coherent events between 0.8 and 1 and a large tail of events with a lower polarization degree. This tail is formed mainly by the events in the lower frequency part of Figure 8 . The region with polarization above 0.8 however constitutes the majority of about $63 \%$ of total cases. This feature will serve in the forthcoming analysis to separate coherent waves which are roughly consistent with the plane wave hypothesis from the whole data set. An indication on the reliability of this procedure is given by a similar analysis of polarization degrees but including only the frequency of the maximum signal in each event, where an increased coherency could be expected. The results of the method by Samson and Olson [1980] are moved

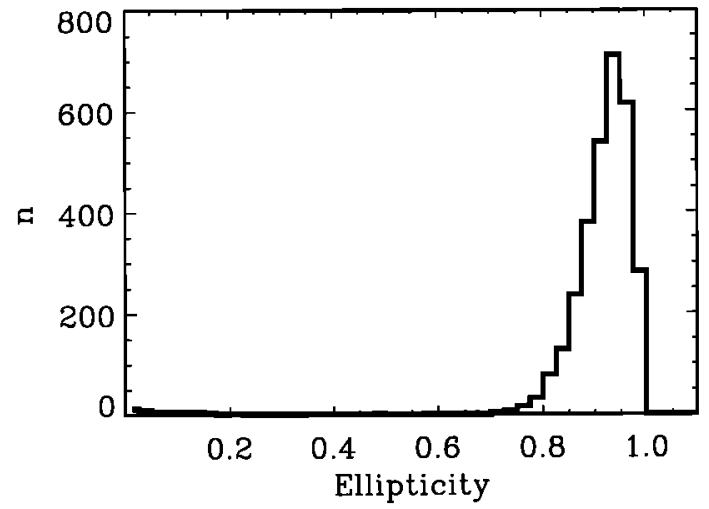

Figure 10. Histogram of the ellipticity for the frequency range $f_{\mathrm{He}^{+}}-0.8 f_{\mathrm{H}^{+}}$except the waves with the polarization degree below 0.8 .

toward higher polarization degrees (a distribution peaked near $P=0.8$ ), and the method of McPherron et al. [1972] places about $87 \%$ of events in the interval $0.8-1$ (not shown).

An assumption on the presence of a single plane wave will be used in further analysis of the wave polarization and propagation. The spectral matrices from the frequency interval $f_{\mathrm{He}^{+}}-$ $0.8 f_{\mathrm{H}}+$ form again the initial data set. This set has been reduced rejecting the matrices with a degree of polarization [McPherron et al., 1972] lower than 0.8 . With this reduced data set, we have first checked the ellipticity and the sense of polarization. A histogram of obtained ellipticities is presented in Figure 10, which shows that the majority of cases has a nearly circular polarization with ellipticities above 0.8 . The analysis of the polarization sense (see section 3 ) provides a clear evidence that all these cases are right polarized. To analyse the major wave propagation properties, a statistics on $\mathbf{k}$ directions can be important. The histograms of the polar angle $\theta$ are given in Figure 11 .

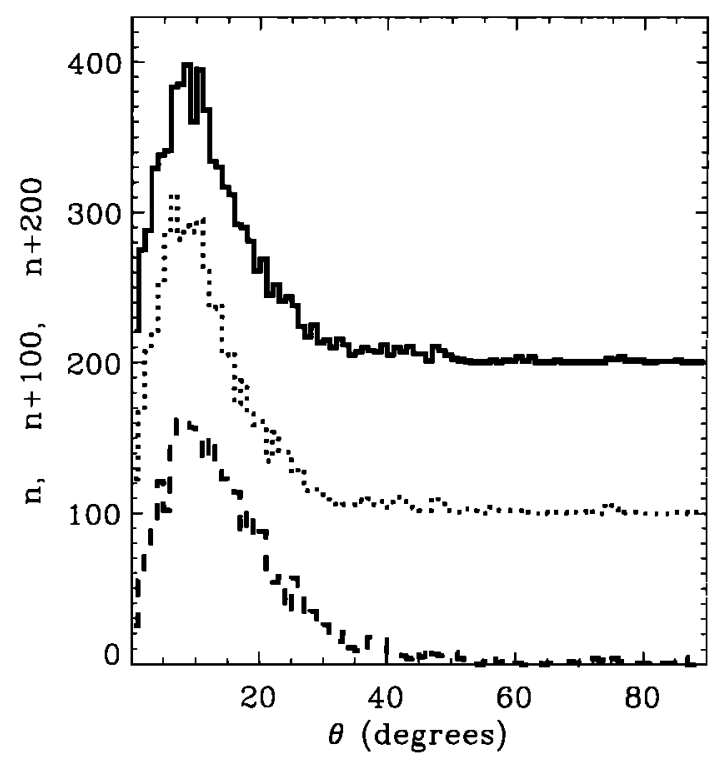

Figure 11. Histogram of the angle deviation $\theta$. (solid line) Method of Means [1972], the number of events in each bin is increased by 200; (dotted line) method of Samson and Olson [1980], the number of events in each bin is increased by 100 ; (dashed line) method of McPherron et al. [1972]. 


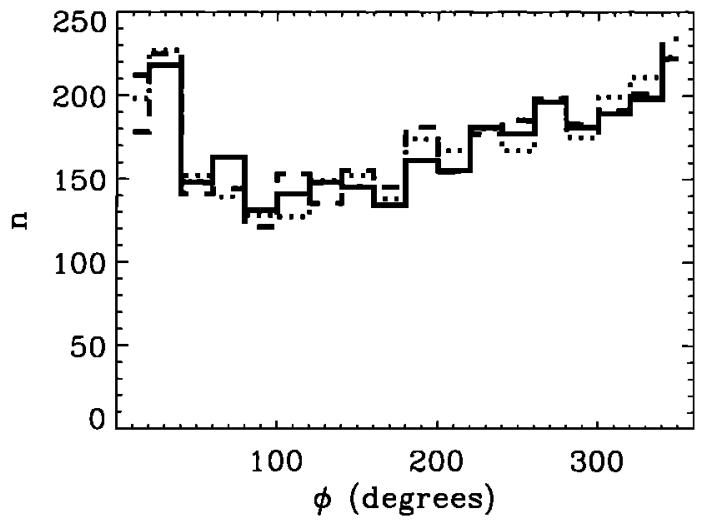

Figure 12. Histogram of the azimuthal angle $\phi$. The direction $\phi=0^{\circ}$ lies in the plane of the local magnetic meridian and points towards lower CGLat. (solid line) Method of Means [1972]; (dotted line) method of Samson and Olson [1980]; (dashed line) method of McPherron et al. [1972].

Three independent methods have been used as in section 3 . The three histograms give very consistent results: a distinct peak with a maximum at $\theta$ of about $8^{\circ}$, a rapidly decreasing number of events for lower $\theta$, and less rapidly fading off for increasing $\theta$ up to $40^{\circ}$. The median values are about $11^{\circ}$ for the methods by Means et al. [1972] and Samson and Olson [1980], and about $13^{\circ}$ with the method by McPherron et al. [1972]. Qualitative properties of the histograms on Figure 11 are reproduced with a similar analysis at frequencies of the maximum signal intensity. A possible dependence of obtained $\theta$ values on the geomagnetic coordinates CGLat and MLT has been checked. Our data set shows no remarkable effect connected with the position of the point of observation. No effect has been also noted concerning the dependence on the normalized wave frequency $f / f_{\mathrm{H}^{+}}$. The second parameter used to describe the $\mathbf{k}$ direction is the azimuthal angle $\phi$ (see Figure 1). Figure 12 shows $\phi$ histograms calculated by the three analysis methods. No distinct peak is seen, and all three methods rather provide a homogeneous coverage of all possible $\phi$ values. A shallow minimum is around $\phi=90^{\circ}$ (roughly the direction of the decreasing MLT) and a slightly enhanced number of events is near $\phi=0^{\circ}$ (direction of the decreasing CGLat). Similar results with no preferred $\phi$ values are also obtained by an analysis at frequencies of the maximum signal intensity. A scatterplot of simultaneously obtained $\theta$ and $\phi$ values proves that both angles are uncorrelated (not shown). The same holds true for the relation of $\phi$ and the wave frequency and also for a dependence on both geomagnetic coordinates CGLat and MLT.

The recognition of upgoing and downgoing waves is needed to complete the description of the wave propagation. A possible way is described in section 2 . Figure 13 shows a histogram of the direction indicator obtained by this procedure. The data set has been further reduced to include only the events where the direction of the electric antenna sufficiently differs from the direction of the background field $\mathbf{B}_{0}$. The criterion was adjusted to have at least $20 \%$ of the total antenna length projected to the plane perpendicular to $\mathbf{B}_{0}$. The analysis gives a distinct peak on negative values of the direction indicator. The exact position and shape of the peak reflect the procedure of the power spectra estimation, but the important result is that only one peak has been obtained. The negative values mean that the waves generally propagate downwards.

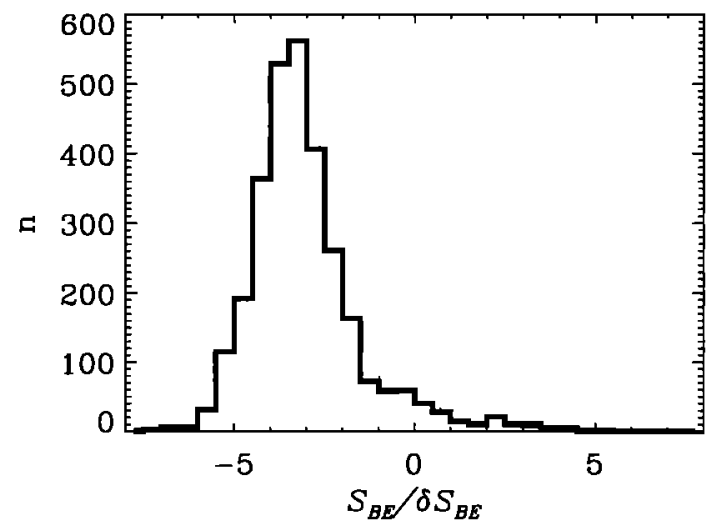

Figure 13. Histogram of the direction indicator. Negative values correspond to downgoing waves, positive values indicate upward propagation.

Although the WDF analysis has not been performed on the whole set of events, a combination of more simple tools provides useful results in this statistical study. It should be noted, that this is only due to special properties of the emissions between $f_{\mathrm{He}^{+}}$and $f_{\mathrm{H}^{+}}$. For the majority of events, the wave propagation can be described by the presence of a single plane wave, which is not a general rule. These favorable conditions also allow to compare the theoretical predictions on the wave mode with the observations. A simple procedure is widely used to examine the dispersion properties of an observed wave field. It uses the Faraday's law, $v_{p}=E_{\perp} / B$, to obtain the phase velocity $v_{p}$ from the measured modulus of the wave magnetic field $B$ and the component $E_{\perp}$ of the wave electric field. The component $E_{\perp}$ is defined as the projection of the electric field vector to the plane perpendicular to the phase velocity. This definition can pose a practical problem connected to the use of the procedure. In a general case two conditions are needed to determine $E_{\perp}$ from the total electric field $E$ : we must know the theoretical dependence of $E_{\perp} / E$ on the angle $\theta$ and the value of $\theta$ itself. Another practical difficulty of this procedure is caused by the validity of the plane wave approximation. If there is a doubt about this hypothesis, the procedure can give an estimation of $v_{p}$ which could not be rigorously interpreted. Both these problems have been removed by the above statistical analysis. Only the data corresponding roughly to the plane wave hypothesis can be selected using the condition $P>0.8$. The $\theta$ histogram reveals a

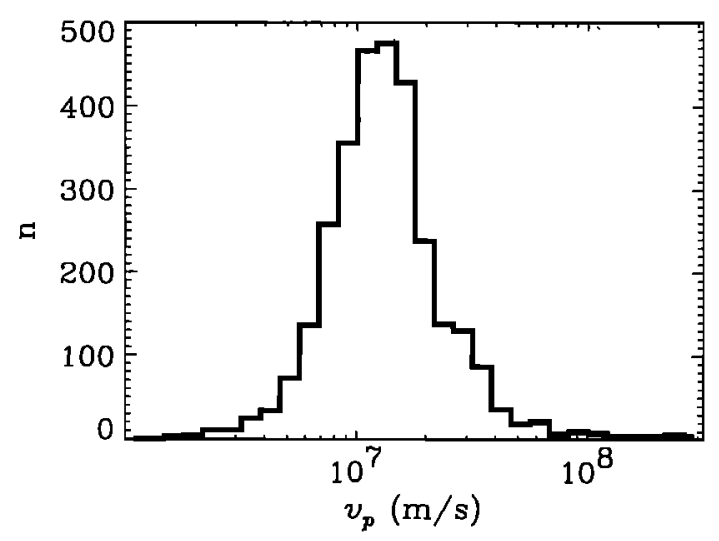

Figure 14. Histogram of the phase velocity modulus estimated from the measurement of the wave electric and magnetic fields. 
nearly parallel propagation with respect to the ambient dc field. Under such conditions, the right-polarized cold plasma mode has the wave electric field perpendicular to the phase velocity, $E_{\perp}=E$.

An additional problem however arises from the real experimental configuration. As noted above, the electric field is measured by a single spinning antenna. The total electric field can be therefore obtained only on a statistical basis. Assume the antenna direction is random and uniformly distributed in the $4 \pi$ solid angle. A mean value of the projection of the total electric field $E$ to the antenna direction will be then $E_{a}=\frac{1}{2} E$ and its standard deviation $\delta E_{a}=\frac{1}{\sqrt{3}} E_{a}$ (about $58 \%$ ). Using these relations, the phase velocity can be estimated,

$$
v_{p}=2 \sqrt{\frac{S_{E_{a}}}{\frac{1}{2} \operatorname{Tr} S_{M}}},
$$

where the factor $\frac{1}{2}$ in front of $\operatorname{Tr} S_{M}$ corresponds to the assumption of a circular polarization, and $S_{E_{a}}$ is the power spectrum of the electric component. Note that only the statistical properties of the antenna direction have been taken into account. This procedure can be therefore justified when the resulting $v_{p}$ values are again examined as a statistical data set.

The Figure 14 presents a result of this approach. The $v_{p}$ histogram shows a distinct peak just above the velocity of $10^{7} \mathrm{~m} / \mathrm{s}$ (the median value is $1.3 \times 10^{7} \mathrm{~m} / \mathrm{s}$ ). The observed peak width corresponds well to the above estimation of the $58 \%$ relative error induced by the spinning antenna. The theory of multicomponent cold plasmas [Smith and Brice, 1964] predicts a value very near to the obtained median for the right-hand-polarized waves below $f_{\mathrm{H}^{+}}$. If we suppose a plasma with $5 \%$ of $\mathrm{H}^{+}$ and $95 \%$ of $\mathrm{O}^{+}$, a proton gyrofrequency of $400 \mathrm{~Hz}$, an electron plasma frequency of $500 \mathrm{kHz}, \theta$ of $10^{\circ}$ and a wave frequency of $0.8 f_{\mathrm{H}^{+}}$, we obtain $v_{p}=0.97 \times 10^{7} \mathrm{~m} / \mathrm{s}$. This value is about inversely proportional to the plasma frequency and ranges from $0.59 \times 10^{7}$ to $1.07 \times 10^{7} \mathrm{~m} / \mathrm{s}$ for wave frequencies between $f_{\mathrm{He}^{+}}$ and $f_{\mathrm{H}^{+}}$. It is also relatively insensitive to the ion composition: a plasma with $50 \%$ of $\mathrm{H}^{+}$increases the theoretical value $v_{p}$ by only about $15 \%$. Taking into account the uncertainties due to the spinning antenna, the experimental values $v_{p}$ well correspond to the cold plasma theory if we consider the average plasma parameters in the low-altitude auroral region.

\section{Discussion and Conclusions}

We have presented an experimental study on electromagnetic waves observed in the low-altitude auroral zone in a large frequency band below the local $f_{\mathrm{H}^{+}}$. The study concerns emissions with a lower frequency limitation similar to the observations of Rauch et al. [1985], and Lefeuvre et al. [1992]. The frequency band is often wider than $f_{\mathrm{H}^{+}} / 4$. The maximum intensity is sometimes below $f_{\mathrm{H}^{+}}$but often no decrease of wave power is observed between these emissions and the associated ELF hiss. The emissions have sometimes a sharp lower cutoff situated between 0.1 and $0.4 f_{\mathrm{H}^{+}}$. A diffuse or fluctuating lower frequency limit is however frequent and can reach up to about $0.7 f_{\mathrm{H}^{+}}$. We have found no direct association of this lower limit with the local $f_{\mathrm{He}^{+}}$. The emissions occur outside the plasmasphere, predominantly at latitudes above $60^{\circ}$. The statistics show a flat maximum of wave power around CGLat of $70^{\circ}$ and does not exclude a possible occurrence also at latitudes above $75^{\circ}$, where we have no data due to the satellite orbit. The MLT distribu- tion reveals the maximum wave power before the local noon, sharp decreases at about 4 hours on the morning side and at 20 hours in the evening. No events have been detected during the local night from 20 to 2 hours. The magnetic power-spectral density currently reaches values of $10^{-6} \mathrm{nT}^{2} / \mathrm{Hz}$ but sometimes the power can reach up to $10^{-3} \mathrm{nT}^{2} / \mathrm{Hz}$. The statistics of the power density show that, on average, the power is a growing function of the wave frequency in all the interval between $f_{\mathrm{He}^{+}}$ and $f_{\mathrm{H}^{+}}$. Although there are cases with the maximum power at lower frequencies, this maximum is significantly more often observed near the upper frequency limit of our analysis.

A majority of cases is highly polarized and we can often assume the presence of a single plane wave. The validity of this hypothesis is also confirmed by the WDF analysis of one example case. Lower values of polarization degree are observed at frequencies below about $0.5 f_{\mathrm{H}^{+}}$, where the statistics give also lower signal intensities. The decreased polarization degree is possibly due to an increased occurrence of complex wave fields in this frequency interval. An example of the WDF reconstruction indicates a simultaneous presence of waves propagating at large $\theta$ and waves with nearly field-aligned wave vectors. Further analysis of the highly polarized emissions shows, that the polarization is always nearly circular and right-handed. This is in agreement with the linear theory of homogeneous cold plasmas, where the medium cannot support left-polarized electromagnetic waves in a frequency interval between $f_{\mathrm{He}^{+}}$and $f_{\mathrm{cL}}$. Our observations are consistent with oxygen-dominated plasmas containing a few percent of protons, where $f_{\mathrm{cL}}$ lies just below $f_{\mathrm{H}^{+}}$. The statistics of wave-vector directions give a downward and nearly field-aligned propagation. The angle $\theta$ between $\mathbf{k}$ and $\mathbf{B}_{0}$ is most frequently around $10^{\circ}$ and values up to $40^{\circ}$ are observed with a strongly decreasing occurrence rate. As it follows from the theoretical cold plasma dispersion, the group velocity direction is even more field-aligned than the wave vector. The azimuth angle $\phi$ measured around the $\mathbf{B}_{0}$ direction is found to be about uniformly distributed, probably due to the prevailing low $\theta$ values. The modulus of the phase velocity, as estimated from the simultaneous magnetic and electric field measurements, is in rough agreement with the cold plasma theory.

As far as we know, only two papers based on Aureol 3 data [Rauch et al., 1985; Lefeuvre et al., 1992] have paid an extended attention to this type of emissions. On the other hand, the narrow-banded emissions as of Temerin and Lysak [1984] have been examined more extensively in the past [e.g., Saito et al., 1987; Gustafsson et al., 1990; Oscarsson et al., 1997]. We believe that the emissions we have examined in the present paper differ in several points trom the narrow-banded cases. (1) The band width, which is easily seen on the spectrograms seems to be a rather unreliable criterion. A more significant one is the simultaneous presence of the electromagnetic ELF hiss. (2) The clear dayside occurrence differs substantially from the maximum occurrence rates in the night hours shown by Saito et al. [1987]. (3) The field-aligned propagation is not directly consistent with the generation mechanism proposed by Temerin and Lysak [1984].

Possible generation processes are discussed by Rauch et al. [1985]. The first is based on the direct generation in the auroral region. At higher altitudes, the waves could be generated at large $\theta$, by a beam of precipitating electrons as in Temerin and Lysak [1984]. During the downward propagation, the waves could experience a refraction leading to a nearly field-aligned propagation. The mechanism of this refraction is however hard. 
to justify and no similar effect has been reported in a recent ray tracing study [Lund and LaBelle, 1997]. The waves propagating at high $\theta$ would rather reflect at the two-ion hybrid frequency $f_{\text {ih }}$ as described by Rauch and Roux [1982]. The value of $f_{\text {ih }}$ is above $0.6 f_{\mathrm{H}^{+}}$for the typical plasma parameters in the auroral region $\left(\mathrm{H}^{+}\right.$fraction below $\left.10 \%\right)$. The observed lower cutoffs are however much lower than $0.6 f_{\mathrm{H}^{+}}$in the majority of our cases. As noted by Rauch et al. [1985], there is also some possibility of direct generation at low altitudes near the satellite, for example, by energetic protons.

We believe that another possible explanation proposed by Rauch et al. [1985] is more promising. It is based on a connection with the electromagnetic ELF hiss observed in the auroral region. During the downward propagation, a part of the hiss can tunnel below the growing two-ion cross-over frequency $f_{\text {co }}$ and stay in the right-hand-polarized mode. The cutoff at $f_{\mathrm{cL}}$ then does not affect these waves. In a homogeneous plasma, this is only possible for the strictly parallel propagation. However, taking into account both the dispersion properties below $f_{\mathrm{H}}$ and the inhomogeneity of the Earth's magnetic field, Le Quéau et al. [1993] and Chust and Le Quéau [1996] found that such a tunnelling is possible in some finite range of non-zero $\theta$ angles. This process could be a possible source of the main part of the observed emissions. It cannot be excluded, that there is also another different generation mechanism which acts together, especially at lower-frequencies below $0.5 f_{\mathrm{H}^{+}}$. We have however observed that the wave intensity mostly grows with the frequency in all the frequency interval up to $f_{\mathrm{H}^{+}}$. This seems to suggest a connection to the ELF hiss above $f_{\mathrm{H}^{+}}$, and to favor the tunnelling of the ELF hiss as the dominating process. Then, the lower frequency limitation at $f_{l}$ could be associated with a mechanism of the hiss generation. Although several studies have been devoted to this topic in the past [e.g., Lefeuvre et al., 1992, and references therein], the origin of the parallel propagating hiss still remains an open question. A probable candidate is the cyclotron resonance with energetic electrons in the equatorial magnetosphere [Thorne et al., 1973]. The lower cutoffs reported in the latter paper are consistent with our observations of $f_{l}$. Another possibility is the Landau resonance with auroral electrons at high altitudes. In this case, the obtained values of $f_{l}$ would correspond to the local lower hybrid frequencies in the generation region. Both processes, however, require a complicated propagation from the source region to obtain parallel propagating waves in the low-altitude auroral zone. A statistical study of the ELF hiss and its association to the emissions below $f_{\mathrm{H}}+$ could be useful to examine some of these speculations.

Our results tempt us to answer some questions raised by Lund and LaBelle [1997]. Their sounding-rocket data reveal an emission between 100 and $250 \mathrm{~Hz}$ attributed to the same wave mode as reported in our study. The authors note a similarity of this observation with "ELF auroral roar" detected on the ground by Sato and Hayashi [1985]. The ray-tracing study of Lund and LaBelle [1997], however, leads to a discrepancy between possible wave-normal directions in the generation region and the mechanism proposed by Temerin and Lysak [1984]. The waves which can reach the ionosphere are more field-aligned than the waves generated by an electron beam. The authors thus conclude that their observation and the "ELF roar" of Sato and Hayashi [1985] could possibly belong to another phenomenon than satellite observations of narrow-banded emissions where the model of Temerin and Lysak [1984] is widely accepted. Our satellite study reports a class of emissions propagating with nearly field-aligned wave-normal directions. However we are far from immediately attributing the observation of Lund and LaBelle [1997] to this class. The contrast between the predominantly dayside occurrence of our events and the nightside case of Lund and LaBelle [1997] could be the first possible objection. The same holds true for the study of Sato and Hayashi [1985]. We hope that future studies will improve the characterization of this, up to now relatively overlooked class of auroral ELF emissions, and give an answer to the open question about its generation mechanism.

Acknowledgments. Many thanks are due to B. Holback (IRFU), the PI of the F4 experiment for the use of his data and for helpful discussions about the data handling. Data are available in France thanks to H. de Feraudy (CETP-UVSQ) who is Co-I of F4. ISDAT (Interactive Science Data Analysıs Tool) developed by A. Lundgien (IRF-U) has been used for the data analysis in this paper The work of $O$. Santolík was partly supported by the Ministère de l'Enseignement Supérieur et de la Recherche (France). He also acknowledges the support of the Czech Grant Agency grants 202/97/1122 and 202/97/P076, and the GAAS grant A3042601.

The Editor thanks Christopher Chaston and another referee for their assistance in evaluating this paper.

\section{References}

Anderson, B.J., R.E. Denton, and S.A. Fuselier, On determining polarization characteristics of ion cyclotron wave magnetic field fluctuations, J. Geophys. Res., 101, 13195-13213, 1996.

Chust, T., and D. Le Quéau, Resonant absorption of downward propagating electromagnetic hiss, J. Geophys. Res., 101, 10695-10710, 1996.

Delannoy, C., and F. Lefeuvre, MAXENTWDF - A computer program for the maximum entropy estımation of a wave distribution function, Comp. Phys. Commun., 40, 389-419, 1986.

Freja Magnetic Field Experiment Team, Magnetic field experiment on the Freja satellite, Space Sci. Rev., 70, 465-482, 1994.

Gurnett, D. A., and T. B. Burns, The low-frequency cutoff of ELF emissions, J. Geophys. Res., 73, 7437-7445, 1968.

Gurnett, D.A., and L.A. Frank, ELF noise bands associated with auroral electron precipitation, J. Geophys. Res., 77, 3411-3417, 1972.

Gustafsson, G., M. André, L. Matson, and H. Koskinen, On waves below the local proton gyrofrequency in auroral acceleration regions, J. Geophys. Res., 95, 5889-5904, 1990.

Holback, B., S.-E. Jansson, L. Ahlen, G. Lundgren, L. Lyngdal, S. Powell, and A. Meyer, The Freja wave and plasma density experiment, Space Sci. Rev., 70, 577-592, 1994.

Lefeuvre, F., and C. Delannoy, Analysis of random electromagnetic wave field by a maximum entropy method, Ann. Telecommun., 34, 204-213, 1979.

Lefeuvre, F., and M. Parrot, The use of the spectral matrix in analysing the propagation characteristics of electromagnetic emissions, Eur. Space Agency Spec. Publ., ESA SP-217, 715-722, 1984.

Lefeuvre, F. Y. Marouan, M. Parrot, and J.L. Rauch, Rapid determination of the sense of polarization and propagation for random electromagnetic wave fields. Application to GEOS1 and AUREOL3 data, Ann. Geophys., 4A6, 457-468, 1986.

Lefeuvre, F., J. L. Rauch, D. Lagoutte, J. J. Berthelier, and J. C. Cerisier, Propagation characteristics of dayside low-altitude hiss: Case studies, J. Geophys. Res., 97, 10601-10620, 1992.

Le Quéau, D., A. Roux, J. L. Rauch, F. Lefeuvre, and J. M. Bosqued, Heating of protons by resonant absorbtion in a multicomponent plasma 2. Theoretical model, J. Geophys. Res., 98, 13363-13375, 1993.

Lund, E.J., and J. LaBelle, On the generation and propagation of auroral electromagnetıc ion cyclotron waves, J. Geophys. Res., 102, 1724117253, 1997.

McPherron, R. L., C. T. Russel, and P. J. Coleman Jr., Fluctuating magnetic fields in the magnetosphere, 2, ULF waves, Space Sci. Rev., 13, 411-454, 1972.

Means, J. D., Use of the three-dimensional covariance matrix in analyzing the polarization properties of plane waves, J. Geophys. Res., 77, 5551-5559, 1972.

Oscarsson, T., and K. Rönnmark, A combined wave distribution func- 
tion and stability analysis of Viking particle and low-frequency data, J. Geophys. Res., 95, 21187-21202, 1990.

Oscarsson, T., A. Vaivads, K. Rönnmark, J. H. Clemmons, H. de Feraudy, and $\mathrm{B}$. Holback, Towards a consistent picture of the generation of electromagnetic ion cyclotron ELF waves on auroral field lines, $J$. Geophys. Res., 102, 24369-24386, 1997.

Parrot, M., and F. Lefeuvre, Statistical study of the propagation characteristics of ELF hiss observed on GEOS-1, outside and inside the plasmasphere, Ann. Geophys., 4, 363-384, 1986.

Pinçon, J. L., Y. Marouan, and F. Lefeuvre, Interpretation of measurements of the polarization percentage for plasma waves, Ann. Geophys., 10, 82-95, 1992.

Rauch, J.L., A. Roux, Ray tracing of ULF waves in a multicomponent magnetospheric plasma: consequences for the generation mechanism of ion cyclotron waves, J. Geophys. Res., 87, 8191-8198, 1982.

Rauch, J.L., F. Lefeuvre, J.C. Cerisier, J.J. Berthelier, N. Boudko, G. Michailova, and O. Kapustina, Attenuation bands and cut off frequencies for ELF electromagnetic waves, in CNES-Results of the ARCAD-3 Project and of the Recent Programmes in Magnetospheric and Ionospheric Physics, p. 435, Cepadues-Editions, Toulouse, France, 1985.

Saito, H., T. Yoshino, and N. Sato, Narrow-banded ELF emissions over the southern polar region, Planet. Space Sci., 35, 745-752, 1987.

Samson, J. C., and J. V. Olson, Some comments on the descriptions of the polarisation states of waves, Geophys. J. R. Astron. Soc., 6I, 115-129, 1980.

Santolík, O., and M. Parrot, The wave distribution function in a hot magnetospheric plasma: The direct problem, J. Geophys. Res., I0I, 10639-10651, 1996.

Sato, N., and K. Hayashi, Band-limited ELF emission burst (auroral roar), J. Geophys. Res., 90, 3531, 1985.

Smith, R.L., and N.M. Brice, Propagation in multicomponent plasmas, J. Geophys. Res., 69, 5029-5040, 1964.
Stix, T. H., Waves in Plasmas, Am. Inst. of Phys., New York, 1992.

Storey, L. R. O., and F. Lefeuvre, Theory for the interpretation of measurements of a random electromagnetic wave field in space, Space Res., 14, 381-386, 1974.

Storey, L. R. O., and F. Lefeuvre, The analysis of 6-component measurement of a random electromagnetic wave field in a magnetoplasma, 1 , The direct problem, Geophys. J. R. Astron. Soc., 56, 255-270, 1979.

Storey, L. R. O., and F. Lefeuvre, The analysis of 6-component measurement of a random electromagnetic wave field in a magnetoplasma, 2 , The integration kernels, Geophys. J. R. Astron. Soc., 62, 173-194, 1980.

Temerin, M., and R. L. Lysak, Electromagnetic ion cyclotron mode (ELF) waves generated by auroral electron precipitation, J. Geophys. Res., 89, 2849-2859, 1984.

Thorne, R.M., E.J. Smith, R.K. Burton, and R.E. Holzer, Plasmaspheric hiss, J. Geophys. Res., 78, 1581-1596, 1973.

Wahlund, J.-E., P. Louarn, T. Chust, H. de Feraudy, A. Roux, B. Holback, P.-O. Dovner, and G. Holmgren, On ion acoustic turbulence and the nonlinear evolution of kinetic Alfven waves in aurora, Geophys. Res. Lett., 21, 1831-1834, 1994.

M. Parrot, Laboratoire de Physique et Chimie de l'Environnement, CNRS, 3A, Avenue de la Recherche Scientifique, 4507I Orléans cedex 02, France. (e-mail: mparrot@cnrs-orleans.fr)

O. Santolík, Faculty of Mathematics and Physics, Charles University, V Holešovičkách 2, CZ-18000 Praha 8, Czech Republic. (e-mail: ondrej.santolik@mff.cuni.cz)

(Received December 18, 1997; revised April 20, 1998;

accepted April 21, 1998.) 